\title{
UNA REFLEXIÓN FILOSÓFICA SOBRE EL ARTE DESPUÉS DE SU CONFUSIÓN CON LA REALIDAD
}

Edgar Vite*

RESUMEN: En este artículo se examinan las circunstancias históricas que produjeron la confusión entre arte y realidad, a partir de los ready-mades de Marcel Duchamp, que se originaron en el arte moderno. El autor analiza la propuesta de Arthur C. Danto para lograr identificar e interpretar este tipo de obras, sustentada en la posibilidad de encontrar un criterio alternativo a la percepción visual, pues resulta insuficiente para distinguir un objeto de uso cotidiano de su homólogo artístico. Danto recurre a ciertos elementos externos de las obras como es su contexto histórico, la forma en que la intención nos orienta en su interpretación y el carácter retórico de las mismas. Sin embargo, su planteamiento conlleva una serie de dificultades, por lo que el autor realiza un balance de las objeciones y críticas más importantes que le han presentado Joseph Margolis y George Dickie.

rose

ABSTRACT: In this paper is studied the origin of the confusion between art and reality, taking as reference the ready-mades of Marcel Duchamp and its origin in Modern Art. The author analyzes the proposal of Arthur Danto to identify and interpret this kind of artworks. This North American philosopher tries to find a criterion, different from the visual perception to distinguish ready-mades from ordinary objects. Danto thinks the answer is in extrinsic elements like the historic context, the intentions of Marcel Duchamp and the rhetoric character of his project. Instead of this his theory has some difficulties, so the author examines the most important objections and critics that have been presented by Joseph Margolis and George Dickie.

PALABRAS ClAVE: Ready-Mades, Arthur Danto, interpretación, retórica.

KEYWORDS: Ready-Mades, Arthur Danto, interpretation, rhetoric.

RECEPCIÓN: 12 de enero 2007.

ACEPTACIÓN: 27 de marzo 2008.

* Departamento Académico de Estudios Generales, ITAM. 
La reproducción total o parcial de este artículo se podrá hacer si el ITAM otorga la autorización previamente por escrito. 


\section{UNA REFLEXIÓN FILOSÓFICA SOBRE EL ARTE DESPUÉS DE SU CONFUSIÓN CON LA REALIDAD}

Pienso en Warhol, que siguió esta línea de investigación con mayor imaginación conceptual que cualquier otro, borrando falsos criterios a cada paso, hasta que empezó a apreciarse que no había nada que no pudiera ser arte. Pero esto ocurría en las artes en todas partes al mismo tiempo - en la danza, en el teatro, en la música. Puesto que cualquier cosa podía ser arte, la cuestión que se planteaba era por qué no todo era arte, esto es, ¿qué diferencia lo que es arte de lo que no lo es?

Arthur C. Danto en La Madona del futuro

\section{$M_{i}$ intención en este artículo es}

reflexionar sobre la confusión entre arte y realidad, que se produjo a partir de los ready-mades de Marcel Duchamp. Con este propósito me interesa retomar el planteamiento de Arthur C. Danto sobre dicho fenómeno, al que denomina "transfiguración del lugar común". Por último, me interesa analizar con detenimiento su propuesta para resolver este problema y presentar un balance de las críticas más importantes que se han presentando a la misma. ${ }^{1}$

${ }^{1}$ Para una revisión más detallada sobre los factores políticos, sociales y culturales que se vieron implicados en la evolución del arte, y especialmente de las artes figurativas, al grado que fue posible que un objeto de uso cotidiano se convirtiera en una pieza artística, consultar Edgar Vite, "Duchamp y el fin de las artes figurativas", en Estudios 82, vol. v, otoño 2007, p. 19-65. 
EDGAR VITE

Los ready-mades son creaciones artísticas que se caracterizan porque no pueden diferenciarse de sus homólogos reales por medio de la percepción. Por esta razón, Danto considera que no es suficiente basarnos en las propiedades estéticas para establecer la distinción entre arte y realidad. Esto se debe a que, tanto la obra de arte, como el objeto de uso cotidiano poseen la misma constitución interna, por lo que no existe una diferencia cualitativa entre ambos y por tanto la belleza resulta un criterio insuficiente:

Sería asombroso que dos cosas tuvieran exactamente la misma forma, tamaño y material, y que una tuviera y la otra careciera de forma significante... Es verdad que cada objeto puede ser situado a la distancia estética más adecuada para su evaluación estética; pero también es factible que la distinción que buscamos pertenezca a otra dimensión ajena a las revelaciones que la distancia estética puede proporcionarnos, y en relación con la cual la distinción entre obras de arte y meras cosas resulta inescrutable. De modo que ninguna de estas teorías nos será de gran ayuda para trazar la línea de demarcación, y mucho menos el hecho histórico de la mera novedad, puesto que cada objeto es discontinuo con todo lo anterior. ${ }^{2}$

En esta medida, Danto plantea la necesidad de establecer un criterio externo que no se sustente en la apariencia y estructura de las obras $y$, por ende, que no se limite a sus características formales. Desde su perspectiva, la manera de alcanzar la distinción entre arte y realidad es por medio de una serie de rasgos sociales, ideológicos y culturales, que constituyen el mundo del arte y que inciden de forma directa en las obras.

El filósofo norteamericano nos presenta un caso hipotético: Pablo Picasso decide sumergir una de sus corbatas en pintura azul, mostrándose en contra de la pictoricidad y las marcas producidas por las pinceladas en el lienzo. Sin embargo, el problema es que un niño también podría tener la idea de pintar una corbata de su padre tal como lo haría Picasso, sin que por ello se convierta en artista:

\footnotetext{
${ }^{2}$ Arthur C. Danto, La transfiguración del lugar común, 2002, Barcelona, Paidós, p. 60.
} 
Estoy dispuesto a ir más lejos e insistir en que, aunque se trata del tipo de indiscernibilidad que nuestros ejemplos requieren, lo que el niño ha realizado no es una obra de arte; algo impide que entre en la congregación de las obras de arte con franquicia, en la que la corbata de Picasso es fácilmente aceptada, aunque sin demasiado entusiasmo. ${ }^{3}$

Para solucionar esta dificultad, Danto propone que toda creación artística muestra los estados internos de su autor y también las circunstancias particulares que afectan dicho proceso. Por esta razón, cuando entramos en contacto con una obra es necesario tomar en cuenta la perspectiva del artista y el modo en que ésta la altera. El niño del ejemplo hipotético no puede ser tratado como un artista, pues no posee el contexto, el desarrollo técnico, ni el conocimiento histórico para producir una obra de arte.

De esta manera, el filósofo norteamericano plantea que la subjetividad resuelve la confusión entre arte y realidad, pues le parece que gracias a ella es posible comprender el modo en que se modifica la estructura de un objeto para dar lugar a algo nuevo. Piensa que al momento de situarnos frente a una pieza artística no debemos olvidar la intención del artista:

Las cualidades de carácter y personalidad que nos hacen tan interesantes como individuos ante los demás - las que nos suscitan esos sentimientos de amor y odio, fascinación y repulsión, y que escapan a cualquier clasificación de distinciones reglamentadas- son los que han definido el problema cuerpo-mente en la tradición filosófica. Es posible suponer que lo que nos interesa en el arte, a la vista de estas estructuras paralelas, es lo mismo que nos importa en lo demás (como si la obra de arte fuera la exteriorización del artista que la ha realizado, como si para apreciar la obra hubiera que ver el mundo a través de la sensibilidad del artistas y no sólo ver el mundo). ${ }^{4}$

Arthur Danto piensa que existen ciertas obras cuyo rasgo distintivo reside en una serie de características que dependen directamente del

${ }^{3}$ Ibid., p. 75.

${ }^{4}$ Ibid., p. 231. 
EDGAR VITE

autor y no se limitan a la estructura material de su trabajo. La distinción entre arte y realidad no se logra a partir de la percepción sensible y, por ello, se requieren de otros elementos que no se vinculen con su apariencia física:

Mi propuesta fue que la diferencia entre obras de arte y meras cosas -entre una pintura roja y un panel pintado de rojo- no puede lograrse viendo a cada uno de los miembros. Es necesario conocer algo acerca del origen del trabajo, cuándo y por quién fue pintado y qué es lo que quiso decir usando una forma tan reducida de hacerlo. De este modo podemos aceptar fácilmente el tipo de explicaciones interpretativas que fabriqué, por el hecho de que ninguna de ellas cambia el modo en que vemos. ${ }^{5}$

En este sentido, plantea que toda obra está relacionada con un marco histórico y una determinada práctica que influyen directamente en la aceptación de un objeto como parte de la esfera artística. Así, se dan una serie de elementos relacionados con el modo en que el contexto altera la forma de producir y comprender el arte. La carga histórica de toda obra se conecta con su carácter intencional y complementa el criterio planteado como alternativa a la percepción:

El que una cosa sea o no una obra de arte y qué tipo de obra sea, si se trata de una, depende por ejemplo de las relaciones entre los cuadros rojos, los artistas, las reglas de significado, las posibilidades históricas y otros elementos similares; algunos de los cuales pueden inferirse de lo que percibimos, incluso si ellos no son percibidos, tales como formas y colores del tipo del paradigma del dolor que fue ilustrado por los clásicos epistemólogos, percibido primitivamente y sin referencia a causas determinadas. ${ }^{6}$

Sin embargo, esta solución al problema de los indiscernibles presenta ciertas complicaciones. Me detendré a analizar algunas de las objeciones presentadas por Joseph Margolis a dicha propuesta. En

${ }^{5}$ Arthur C. Danto, "Indiscernibility and Perception: A reply to Joseph Margolis", en British Journal of Aesthetics, Oxford, vol. 39, n 4, October, 1999, p. 325.

${ }^{6}$ Ibid., p. 326. 
primer lugar, considera que al rechazar la percepción como un criterio adecuado, Danto se sitúa en otro extremo y piensa que la distinción no se relaciona con las propiedades intrínsecas del objeto, sino con un tipo de cualidades que dependen exclusivamente del contexto histórico y de la intención del artista:

Por contraste, Danto dice que no existen diferencias perceptuales (sensorialmente discernibles) entre una obra de arte y una 'mera cosa' (algo que no es una obra de arte) o entre dos obras de arte diferentes; e inclusive llega a afirmar que el único tipo de diferencias que existen entre ese par de cosas no es de orden sensible, sino que corresponde a la historia (en particular de la historia de la producción) y la intención del artista. $^{7}$

Al descartar la percepción sensible para resolver la mencionada confusión, termina estableciendo que las obras no existen como objetos reales, sino que se convierten en arte bajo ciertas circunstancias, en las que el autor los reviste de un nuevo significado, dotándolos de una nueva identidad. Por lo tanto, desde la perspectiva de Margolis, Arthur Danto da lugar a una fuerte escisión entre arte y realidad, pues de su postura se sigue que las obras de arte no existen como una clase de cosas dentro del mundo: "aparentemente para Danto, sólo por medio de la imputación retórica de ciertos atributos indiscernibles estamos justificados a tratar las meras cosas (cosas que no son obras de arte) como obras de arte. Realmente no existe nada como obra de arte."

En mi opinión, esta crítica no toma en cuenta que Danto no se refiere a todas las obras de arte, sino a aquellas en las que se genera la mencionada confusión. Por lo tanto, es necesario considerar que su preocupación se centra en aquellas obras que causan un efecto desconcertante y nos obligan a cuestionarnos si lo que tenemos enfrente realmente es arte. En el fondo, me parece que lo que hace es cuestionar la existencia de una percepción neutral, pues existen una

${ }^{7}$ Joseph Margolis, "Farewell to Danto and Goodman", en British Journal of Aesthetics: Oxford, vol. 38, n 4, October 1998, p. 354.

${ }^{8}$ Ibid., p. 365. 
EDGAR VITE

serie de elementos externos que interfieren al momento de contemplar una obra de arte.

En esta medida, el filósofo norteamericano plantea que pueden existir dos cosas tan similares que, a pesar de ser indiscernibles, sensiblemente son distintas por la intención del sujeto que las produce. En su opinión la intención del artista presenta tal fuerza que es capaz de cambiar el modo en que entendemos la realidad y, sobre todo, la manera en que comprendemos un objeto que forma parte de ella. De esta manera la intencionalidad nos permite descubrir nuevos significados en los objetos a los que ya estamos acostumbrados:

Ver un objeto, y ver un objeto al que la interpretación transforma en una obra, son cosas claramente distintas, por mucho que la interpretación devuelva el objeto a sí mismo al decir que la obra es el objeto. ¿Pero qué tipo de identificación es ésta? Por el carácter constituyente de la interpretación, el objeto no era una obra hasta que se convirtió en una de ellas. Como procedimiento transformativo, la interpretación es algo parecido al bautismo, no en el sentido de dar un nuevo nombre, sino una nueva identidad, una participación en la comunidad de elegidos. ${ }^{9}$

Al respecto, podemos remitirnos a la conocida Fuente de Marcel Duchamp, quien al nombrar de este modo su obra, sugirió realizar una interpretación distinta sobre este objeto y por lo tanto mostró que un urinario también puede convertirse en una obra de arte. Independientemente del carácter irónico de este trabajo, podemos percatarnos que Duchamp nos hace revalorar algo que nunca antes hubiéramos tomado por asombroso o interesante. Por esta razón, el artista francés no se preocupa por la carga descriptiva de su obra, sino que se centra en el modo en que su interpretación la afecta:

La identificación de este objeto como una fuente no es una clasificación, sino una interpretación: el hecho de decir que un urinario es una fuente, es un fenómeno que en otro lugar he nombrado como identificación artística, la cual se refiere a que este 'es' en cuestión resulta

${ }^{9}$ Arthur C. Danto, La transfiguración ..., op. cit., p. 185. 
consistente (pero solamente consistente) con la falsedad literal de su identificación. ${ }^{10}$

A partir de esta obra, Danto establece que la interpretación por sí sola transforma un objeto determinado, pues al brindar una nueva lectura, sería posible distinguirlo de su homólogo artístico. Por esta razón, cuando decimos que Fuente es una obra de arte no existe una relación de identidad entre el objeto y el nombre, sino una relación interpretativa. Para Danto la interpretación en el arte no sólo implica una explicación de la obra, sino una forma de transfiguración: "Mi teoría sobre la interpretación es constitutiva, esto se debe a que un objeto es una obra de arte solamente en relación a una interpretación. Podemos explicar esto desde una perspectiva lógica. La interpretación en mi opinión es transfigurativa. Transforma los objetos en obras de arte y de ella depende la atribución de la identificación artística."11

Por todo lo anterior se vuelve necesario analizar algunos conceptos que se vinculan con la forma en que la visión particular del artista configura su obra y nos brinda una serie de pautas para apreciarla y comprenderla mejor. Es necesario detenernos a analizar el carácter retórico que, en opinión de Danto, posee toda creación artística. Este aspecto se refiere al hecho de que el creador al producir una obra, la dota de una serie de características muy particulares con la finalidad de transmitir un mensaje. ${ }^{12}$ El mensaje que nos presenta el artista no es

${ }^{10}$ Arthur C. Danto, The Philosophical Disenfranchisement of Art, 1986, New York, Columbia University Press, p. 41.

${ }^{11}$ Ibid., p. 44-5.

${ }^{12}$ Por tanto la finalidad del retórico consistiría en producir una determinada actitud en su audiencia, sin que se dé cuenta, o al menos sin que resulte algo obvio. En este sentido, el retórico se sirve de una serie de habilidades para lograr convencer y también para producir una reacción en sus espectadores. En esta medida, el artista presenta una cierta intención retórica, pues pretende producir una emoción en el espectador, no en el sentido de una manipulación, sino porque busca que su mensaje sea asimilado de una forma determinada: "Para un retórico no basta con demostrar que cierto sentimiento debieran producirse, o que estaría justificado que tú (su auditorio) sintieras, y quizás injustificado que no sintieras: lo único que sabe el retórico es que será digno de tal nombre si logra que tengas dicha emoción (sin decirte lo que has de sentir). De alguna manera prodigiosa habrá de apoderase de la conciencia y 
EDGAR VITE

neutral, sino que está configurado de una forma para lograr un cierto efecto en el público:

Como tal, su función es la de provocar en el auditorio cierta actitud hacia el objeto de su discurso: hacer que el tema sea visto bajo cierta luz. Este incremento suplementario de una actividad que va más allá de la estricta comunicación de los hechos es sin duda lo que hace parecer a la retórica manipuladora, al retórico insincero y, en general, exagerado a todo lo 'retórico'. Es cierto que el retórico -y cualquiera de nosotros entregado a una estrategia retórica- no se limita a afirmar hechos, sino que los sugiere de tal manera que moldea la forma en que el auditorio los percibe: carece de interés lógico que el retórico se sitúe en un nivel comunicativo factual; partimos del presupuesto de que los hechos -a los que a menudo se nos invita a ceñirnos- se dan por sentados, y es a partir de esta asunción que la retórica interviene. ${ }^{13}$

En esta medida la relación entre el espectador y el creador es complementaria. El mensaje no adquiere su sentido pleno hasta que no entra en contacto con la audiencia y la obra es experimentada por otro. El arte se convierte en una especie de acertijo que permanece cerrado hasta que entra en contacto con el público y es resuelto. La interpretación se constituye como un tránsito constante entre lo que quiere decir el autor y lo que entiende el espectador; el arte da lugar a la especulación y a la imaginación del auditorio, por lo que dicho ejercicio comunicativo se mantiene abierto y no implica una sola respuesta.

Así, llegamos a la noción de metáfora, que se desprende de la relación entre arte y retórica. Danto se interesa en mostrar cómo es posible distinguir entre una representación metafórica y una simple representación para solucionar el problema de los indiscernibles.

llevarla al estado que pretende. No trata con autómatas ni con seres puramente racionales. Por eso la retórica es el arte de la persuasión y de la lógica, y en tanto que vertiente psicológica del arte de la demostración, debe saber tanto captarse a la audiencia como caracterizar los hechos y sus interrelaciones." Cfr. Arthur C. Danto, La transfiguración del lugar común, 2002, Barcelona, Paidós, p. 245.

${ }^{13}$ Arthur C. Danto, ibid., p. 240. 
Piensa que el arte presenta un fuerte carácter simbólico, lo que permite diferenciarlo de otro tipo de representaciones:

La cuestión puede plantearse como sigue: ¿por qué la diferencia entre un retrato de Napoleón como emperador romano y un cuadro en que Napoleón posa como modelo de un emperador romano no es sólo una diferencia de contenido? Y si fuera sólo una diferencia de contenido, ¿por qué usar una metáfora que muestra a Napoleón como una figura de la grandeur imperial en lugar de limitarse a mostrar a Napoleón entre los fastos de la grandeur imperial que, como sabemos, lo rodeaban con profusión? ¿Por qué no "dejar a los hechos hablar por sí mismos”, tanto más cuando la metáfora en sí no aporta ningún hecho nuevo? Esto nos devuelve a la pregunta sobre el sentido de la propia metáfora. ${ }^{14}$

Esto lleva a Danto a afirmar que la intencionalidad en el arte no puede reducirse a una simple representación, sino que se trata de una representación metafórica. En esta medida le parece que el espectador se encarga de reconectar el símbolo y desentrañar el significado oculto de la obra. La tarea del espectador no puede limitarse exclusivamente a la contemplación, no puede reducirse a una postura pasiva, sino que debe involucrarse y participar activamente para completar la experiencia artística:

Así la obra de arte se constituye como una representación tout court; estoy convencido de que esto es cierto de las obras de arte, y en general, de las representaciones, ya se logre conscientemente -como en el espectro de obras que hemos analizado-, o de una manera más ingenua, cuando simplemente resulta que el artista dota a su tema de inesperados pero penetrantes atributos. Entender la obra de arte es captar la metáfora que -me parece- siempre hay en ella. ${ }^{15}$

En la creación literaria encontramos un fenómeno análogo, pues la habilidad y creatividad de un escritor reside en su uso del lenguaje, en su capacidad de construir nuevos mundos, a partir de lo que cono-

${ }^{14}$ Ibid., p. 243-4.

${ }^{15}$ Ibid., p. 248. 
EDGAR VITE

cemos y la forma usual en que nos comunicarnos. En este sentido, un buen escritor es el que es capaz de jugar con el lenguaje, con la ambigüedad, la plurisignificación y la connotación, dando lugar a una serie de nuevas posibilidades. ${ }^{16}$ Ocurre algo muy similar en el arte, sobre todo en lo que se refiere a las artes figurativas, en las que existe una fuerte carga metafórica. En este sentido, la teoría de la transparencia en el arte no da cuenta de la diversidad de obras artísticas, sobre todo de aquellas cuya fuerza significativa no se reduce a la inmediatez de la percepción:

Por ahora sólo haré notar que "el agua está hirviendo" equivale a precisar que "el agua ha alcanzado los cien grados" (si bien no podemos precisar tanto para sustituir "le hervía la sangre": el sujeto del enunciado habría muerto por combustión interna). En general es característico de las metáforas resistirse a tales precisiones y sustituciones, y la explicación de esto quizás podría darnos la clave de este concepto. Debería estar al menos intuitivamente claro que hay clichés pictóricos, como hay metáforas pictóricas, pero no todas las pinturas son una cosa u otra. A alguien se le ocurrió poner estrellas y líneas curvas sobre la cabeza de un personaje como metáfora de haberse dado un buen golpe, lo cual se ha convertido en un cliché en la notación de las historietas: ¿es su equivalente verbal "ver las estrellas" o el otro es su equivalente pictórico? Pero estrictamente una imagen de un hombre con estrellas sobre la cabeza sólo puede ser la imagen de un hombre con estrellas sobre al cabeza. ${ }^{17}$

${ }^{16}$ Por tanto se vuelve necesario distinguir entre dos tipos de metáforas: aquellas que se consideran vivas y las denominadas muertas. Para Danto, el lenguaje se mueve en ambos registros, de manera que el segundo corresponde a su literalidad y el primero se refiere a su riqueza estética. De manera que no podemos considerar que todas las manifestaciones lingüísticas, igual que en el arte, presenten un mensaje oculto, aunque algunas sí lo hacen. Por tanto se vuelve indispensable distinguir entre ambas funciones comunicativas: "La teoría de que las metáforas son expresiones desviadas reconoce - me parece- lo que yo trataba de argumentar: ha de haber alguna distinción que sirva para separar las metáforas jóvenes y las viejas de las expresiones literales (que no tienen el mismo ciclo vital), cosa que el elegante concepto de desviación hace estructural y magníficamente. La desviación deberá distinguirse de la deformidad o de la agramaticalidad, sin tener nada que ver con ninguna consideración estadística." Cfr. Arthur C. Danto, ibid., p. 255.

${ }^{17}$ Ibid., p. 255. 
Danto plantea que existe una clara diferencia entre un discurso literal y uno intensivo. El primero se caracteriza porque el mensaje y su forma resultan claros y denotativos, a diferencia del intensivo en el cual se da lugar a una multiplicidad de interpretaciones, pues el mensaje está cargado de una gama de sentido y por tanto da lugar a diversas lecturas:

Me parece que no sería prudente ir más allá de este punto. Sería una agradable distracción ejercitar la semántica de algunos tropos retóricos, pero esto puede dejarse para que practiquen los eventuales entusiastas (o detractores) de la teoría. Desde mi punto de vista, me conformo con haber mostrado que las metáforas encarnan algunas de las estructuras que he supuesto en la obra de arte: no se limitan a representar objetos, sino que las propiedades del modo de representación mismo pueden ser un elemento para entenderlas. Al fin y al cabo es un lugar común que cada metáfora es como un pequeño poema. A decir por los rasgos que hemos identificado, las metáforas son pequeñas obras de arte. ${ }^{18}$

Joseph Margolis cuestiona el modo en que Danto presenta la predicación en la esfera artística. Desde su perspectiva, éste plantea que la nominación de algo como una obra de arte radica en su oposición a la realidad; esto significa que los atributos artísticos le vienen dados gracias a la habilidad retórica de su autor, mediante la que redefine los objetos que nos rodean. Por esta razón la producción artística depende de la manera en que el autor transforma lo existente por medio de su creatividad e imaginación, aún cuando no altera sus propiedades materiales:

Él no sostiene, por ejemplo, que los atributos representacionales o expresivos usualmente adscritos (no impuestos) a las obras de arte se reduzcan a atributos materiales. No, esas propiedades solamente son adscritas imaginativamente, no son propiedades reales, son claramente análogas (tal vez más que análogas) a las atribuciones que hacemos sobre nosotros mismos. ${ }^{19}$

\footnotetext{
${ }^{18}$ Ibid., p. 270.

${ }^{19}$ Joseph Margolis, op.cit., p. 367.
} 
EDGAR VITE

Sobre este aspecto encontramos la propuesta de George Dickie, quien considera criticable que un objeto determinado se convierta en una obra de arte cuando se le denomina como tal, al existir una serie de factores por los que conscientemente el creador dice: "Esto es una obra de arte." Por lo tanto, desde esta perspectiva algo sería una obra de arte debido a su predicación como tal y, de este modo, se distinguiría del resto de la realidad:

Danto aclara su intención al afirmar que el "arte es un lenguaje de clases, en el sentido, al menos, de que una obra de arte dice algo". Asumiendo que 'dice algo' significa 'hace una declaración', es, según Danto, una condición necesaria del arte. Esta conclusión supone claramente que la posición de Danto es la tercera a la que nos hemos referido, es decir, para que algo sea una obra de arte es necesario que esa cosa esté a cierta distancia de a realidad siendo una declaración. ${ }^{20}$

Para Dickie la mera enunciación o señalización de un objeto como una obra de arte resulta un criterio insuficiente. Esto se debe a que existe una diferencia radical entre hacer arte y declarar que se está haciendo arte, por lo que el establecimiento de algo como una pieza artística no puede equipararse con una especie de bautismo, sino que resulta indispensable establecer otro tipo de condiciones por las que un objeto adquiere ese estatus. ${ }^{21}$

Por esta razón, un aspecto paradójico del planteamiento de Danto consiste en que, si basamos la diferencia exclusivamente en la habilidad retórica y la intencionalidad, cualquiera que desarrolle dichas capacidades podría ser tomado por artista. La intención es un criterio muy polémico para establecer lo que es arte y diferenciarlo de lo que no lo es, pues no siempre es posible conocer lo que un artista se propone con su obra. Si consideramos que la voluntad es suficiente para transformar cualquier cosa en arte, dicho criterio produce una clara desconfianza, pues la libertad de escoger y decidir no es exclu-

${ }^{20}$ George Dickie, El círculo del arte, 2005, Barcelona, Paidós, p. 37-8.

${ }^{21}$ Ibid., p. 91. 
siva de la esfera artística, sino que corresponde al resto de los seres humanos por igual. ${ }^{22}$

Margolis sugiere que el principal error del planteamiento de Danto consiste en separar la percepción como parte esencial de nuestra relación con la esfera artística y, en lugar de esto, presentar una serie de criterios externos que no tienen nada que ver con la constitución interna del objeto. Aquí el problema radica en no tomar en cuenta que la contemplación de las obras de arte ya involucra una forma de comprenderlas; por esta razón, no tiene sentido separar las propiedades sensibles de los rasgos conceptuales, correspondientes a todo trabajo artístico:

La percepción sensorial, siempre está determinada con elementos conceptuales de este tipo (inclusive si no está establecido específicamente lo que se requiere para captar el Warhol propiamente). No existe percepción sensorial independiente, a menos que acordemos abstraernos del entramado cultural al que estamos acostumbrados utilizar cuando percibimos algo. ${ }^{23}$

Para Margolis es imposible establecer la existencia de un modo neutral de percepción, pues toda forma de ver está influenciada por una serie de rasgos ideológicos y culturales que son inseparables. A pesar de esto, le parece que lo rescatable de la propuesta de Danto radica en el énfasis que pone en el arte como una práctica, que involucra una serie de factores que van más allá de la experiencia sensible:

La solución de Danto requiere que el contexto el cual es asignado a las 'cosas reales', esté acompañado intencionalmente por propiedades culturales, establecidas por individuos culturalmente aptos, quienes habitan en un mundo intencionado. Ya he ventilado mi objeción frente

${ }^{22}$ En este sentido, me parece necesario considerar que el peso que Danto le da al autor no puede tomarse del mismo modo en cualquier corriente y época artística. Esto se debe a que la finalidad del individuo por establecerse a sí mismo como la piedra de toque en los movimientos sociales y culturales no corresponde a cualquier etapa, sino que es propia de las vanguardias artísticas; posteriormente dicho gesto fue retomado por el arte contemporáneo.

${ }^{23}$ Joseph Margolis, op. cit., p. 371. 
EDGAR VITE

la afirmación de un realismo humano y la negación de un sustento real para cualquier discurso, acción o arte desarrollado por los hombres. Una alternativa a la teoría de Danto puede ser la siguiente: las acciones son reales, están determinadas por movimientos físicos, involucran una serie de prácticas culturales de nuestra sociedad y como resultado realmente poseen y exhiben propiedades intencionales. ${ }^{24}$

En mi opinión, ésta es una de las críticas más fuertes de Margolis a la teoría de Danto, pues se centra en un hecho que es común a toda obra y esto consiste en que la percepción ya implica una forma de ver; por ello, no es necesario dejar de lado las propiedades físicas de las creaciones artísticas, sino más bien considerar que el carácter cognitivo del arte está determinado por una serie de elementos externos que determinan nuestra forma de apreciarlo. El filósofo norteamericano no es ajeno a esto, pues en su noción sobre la interpretación del arte encontramos una serie de factores externos, que influyen directamente en nuestra percepción de las obras.

Sobre este punto Danto plantea que, de acuerdo a cada época, poseemos una serie de creencias y hábitos que nos permiten clasificar y comprender un objeto dentro de la esfera artística. Piensa que Margolis se equivoca al establecer que la percepción solamente está determinada por elementos internos, pues existen ciertos casos en los que la forma de ver se altera por la intención y explicación del artista; el modo de discernir entre un objeto y su homólogo artístico depende de la manera en que la práctica artística altera los criterios con los que estamos acostumbrados a enfrentarnos a las diversas manifestaciones:

El primer cuadro negro del que tuve conocimiento estaba impreso en Tristam Shandy, y Sterne da al lector lo que es necesario para entender por qué aparece ahí. De otro modo el lector, a pesar de que posea un gran bagaje cultural, no lo percibiría más que como un simple cuadro negro. Sterne explica cómo es posible que se trate de un cuadro negro carente de significado. En esta medida hubiera sido prácticamente imposible encontrar obras de arte como los cuadros monocromos antes de

${ }^{24}$ Ibid., p. 374. 
$1869[\ldots]$ En nuestra percepción de cualquier cosa, especialmente del arte, un gran número de creencias culturales, las cuales interfieren en la forma en que pensamos sobre lo que vemos, hasta que por alguna razón cometemos un error y descubrimos que alguna de dichas creencias falla en un caso determinado. ${ }^{25}$

De este modo, Danto establece que requerimos de dichos elementos para comprender e interpretar el arte adecuadamente, por lo que hubo un momento en el que fue necesario cambiar la mentalidad de un grupo para que aceptara un determinado objeto como parte del círculo artístico. Por esta razón, piensa que se requiere de una explicación que no se restrinja a las propiedades materiales de las obras y que permita una comprensión más completa del arte.

La percepción y la intención por sí solas son insuficientes para clasificar algo como una obra de arte, por lo que es necesario tomar en cuenta el contexto histórico que, al producir un cambio de paradigma, altera nuestra forma de interpretación. Desde mi perspectiva, Arthur Danto no niega el lugar de la percepción al momento de entrar en contacto con las obras de arte, sino más bien afirma que es un criterio ineficaz para explicar casos difíciles, como los ready-mades. Finalmente, debo concluir que la evolución de las obras nos muestra la vigencia de la reflexión filosófica en torno a los criterios que nos permiten identificar y comprender el arte.

${ }^{25}$ Arthur C. Danto, "Indiscernibility and Perception: A reply to Joseph Margolis", op. cit., p. 327. 
La reproducción total o parcial de este artículo se podrá hacer si el ITAM otorga la autorización previamente por escrito. 